I) Bei sieben photometrisch beobachteten kleinen Planeten zeigen sich Helligkeitsänderungen, die in Zusammenhang mit der Phase zu stehen scheinen.

2) Auf diese Helligkeitsënderungen lässt sich das Lambert' sche Phasengesetz nicht anwenden.

3) Die untersuchten Planeten lassen sich in zwei Gruppen theilen. Bei der einen, welche Vesta, Iris, Massalia und Amphitrite umfasst, sind die Helligkeitsänderungen nur in der Nâhe der Opposition merklich, während bei der anderen, welche von Ceres, Pallas und Irene gebildet wird, die Aenderungen ziemlich gleíchmässig über den ganzen Verlauf der Phasenänderung vor sich zu gehen scheinen.
4) Die Planeten der ersten Gruppe zeigen in ihrem Verhalten absolute Uebereinstimmung mit dem Planeten Mars; es ist daher wahrscheinlich, dass sie auch dieselbe oder wenigstens sehr ähnliche physische Beschaffenheit besitzen wie dieser.

5) Bei den Planeten der zweiten Gruppe, bei denen die Resultate im Allgemeinen etwas weniger sicher $z u$ sein scheinen, lässt sich eine gewisse Aehnlichkeit der Lichtcurve mit der für den Mond bestimmten erkennen; noch besser scheint ihr Verhalten übereinzustimmen mit dem Planeten Merkur, und es ist daher nicht unwahrscheinlich, dass sie in Bezug anf ihre physische Beschaffenheit mit diesem die meiste Verwandtschaft haben.

Potsdam, Astrophysikalisches Observatorium, 1886 März I4.

\title{
Observations of Minor Planets
}

made at the U. S. N. Observatory, Washington, with the 9.6 inch Equatorial, by Prof. E. Frisby. (Communicated by Commodore Geo. E. Belknap, U. S. Navy, Superintendent.)

\begin{tabular}{|c|c|c|c|c|c|c|c|c|c|c|}
\hline 1885 & Wash. M.T. & $\Delta \alpha$ & $\Delta \delta$ & Cp. & $\alpha$ app. & $\log p .4$ & $\delta$ app. & $\log p .4$ & Red.ad 1.app. & $*$ \\
\hline \multicolumn{11}{|c|}{ (24I) Germania. } \\
\hline $\mathrm{n}$. & $8^{\mathrm{h}} 16^{\mathrm{m}} 57^{\mathrm{s}}$ & $+1^{\mathrm{m}} 4^{\mathrm{s}} \cdot 47$ & $+4^{\prime \prime} 34^{\prime \prime} .2$ & $15 \cdot 3$ & $\mathrm{O}^{\mathrm{h}} \mathrm{I} 7^{\mathrm{m}} 3^{2^{\mathrm{s}}} .09$ & $9 \cdot 5$ & $+7^{\circ}$ & 0.689 & $7+$ & \\
\hline 10 & $6 \cdot 350$ & $-2 \quad 0.06$ & $\begin{array}{ll}\text { I I } & 9.7\end{array}$ & 11.4 & 02045.34 & 9. & +73345.7 & 0.667 & $+0.07+0.7$ & \\
\hline I 3 & $\begin{array}{lll}6 & 19 & 56\end{array}$ & +111.08 & +420.0 & 20.4 & 0246.45 & $9.25^{\circ}$ & +749 I5.3 & $0.76 \mathrm{I}$ & $+0.04+0.6$ & \\
\hline eb. & 8 I 224 & +26.30 & +1454.9 & I $5 \cdot 3$ & $\circ \quad 49 \quad 47.74$ & $9.66 \mathrm{I}$ & +95459.9 & 0.717 & $-0.06-1.5$ & \\
\hline 16 & 73942 & $+035 \cdot 3^{2}$ & +014.8 & 10.2 & $1718.3^{2}$ & 9.529 & +11236.3 & 0.685 & $-0.10-1.9$ & \\
\hline
\end{tabular}

(33) Polyhymia.

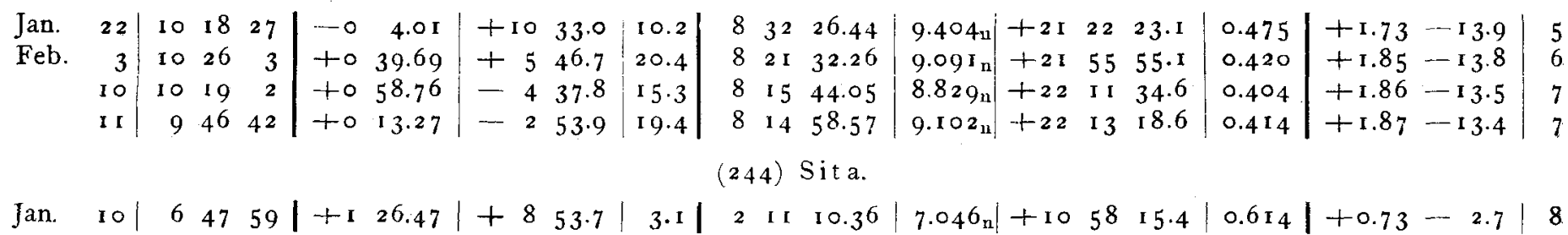

(56) Melete.

\begin{tabular}{|c|c|c|c|c|c|c|c|c|c|c|c|c|c|c|c|c|}
\hline \multirow[t]{2}{*}{. } & I I & 43 & 17 & +037.54 & +3 & 34.9 & $15 \cdot 3$ & Io & $1 \mathrm{I}$ & 35.45 & $9.094 \mathrm{n}$ & +2 & 25 & $3^{2.6}$ & 0.719 & $+1.82-15.7$ \\
\hline & IO & 25 & 27 & 9.25 & +8 & 37.5 & $17 \cdot 4$ & Io & 10 & 48.67 & $9.410_{n}$ & +2 & 30 & 35.2 & 0.716 & $+1.83-15.7$ \\
\hline rch & I 2 & I 4 & 28 & $-33^{6.5} \mathrm{I}$ & 2 & 26.8 & 10.2 & 9 & 49 & $13.5^{I}$ & 9,280 & +5 & I 8 & $4^{8.7}$ & $0.69^{2}$ & $+1.85-16.0$ \\
\hline Io & 9 & 7 & 50 & to 26.11 & - & 22.1 & 19.4 & 9 & 48 & 33.97 & $9.224 \mathrm{n}$ & +5 & 24 & $34 \cdot 5$ & 0.690 & $+1.86-16.1$ \\
\hline 14 & 10 & 8 & 25 & +131.04 & +6 & 0.5 & $15 \cdot 3$ & 9 & 45 & 49.90 & $8.070_{n}$ & +5 & $5 \mathrm{I}$ & 12.3 & $0.68 \mathrm{I}$ & $+1.85-16.1$ \\
\hline
\end{tabular}

(59) Elpis.

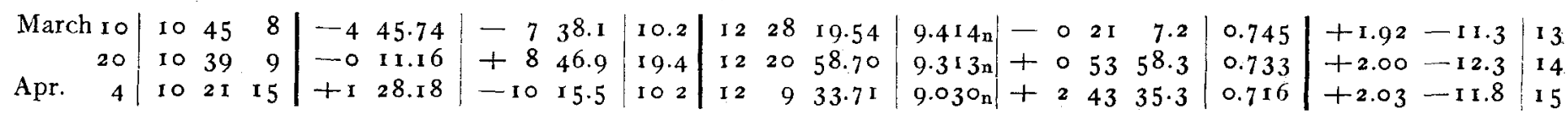

(62) Er a tho.

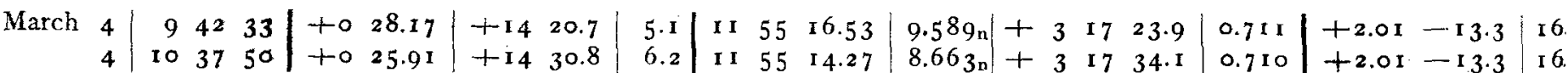




\begin{tabular}{|c|c|c|c|c|c|c|c|c|c|}
\hline 1885 & Wash. M.T. & $\Delta \alpha$ & $\Delta \delta$ & Cp. & $\alpha$ app. & $|\log p .4|$ & $\delta$ app. & $\log p . A$ & Red.ad 1. app. \\
\hline
\end{tabular}

(186) Celuta.

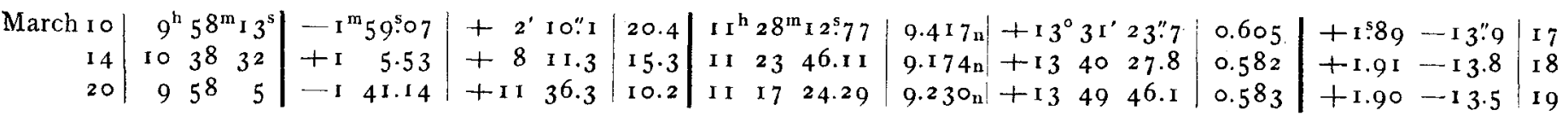

(37) Fides.

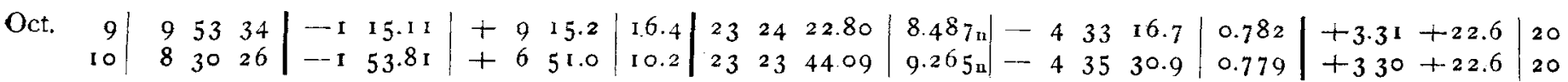

(7 I) $\mathrm{N}$ io be.

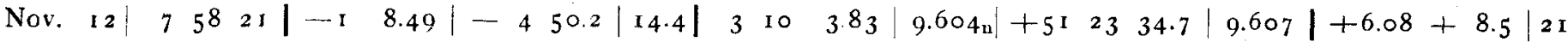

(185) Eunike.

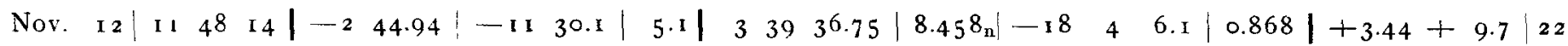
(221) EOs

Nov. I $4|95931|-0 \quad 0.88|+105.3| 20.4|34858.21| 9.399_{n}|+32524.8| 0.713|+3.86+12.4| 23$ (209) 1)ido.

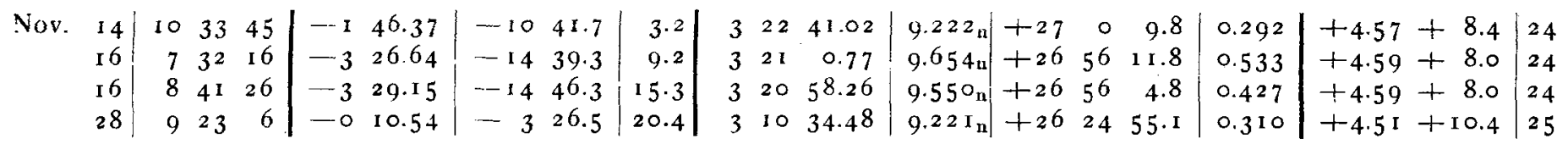
(241) Germania.

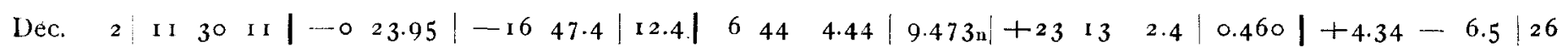
Mean Places of Comparison Stars for 1885.0.

\begin{tabular}{|c|c|c|c|c|c|c|c|c|}
\hline * & $\alpha \leq 885.0$ & $\delta$ I 885.0 & Authority & * & $\alpha 18$ & 85.0 & $\delta 1885.0$ & Authority \\
\hline $\mathbf{I}$ & $0^{h} 16^{m} 27^{s} \cdot 55$ & $+7^{\circ} 14^{\prime} 4^{\prime \prime} .8$ & $W_{1} 0^{h} 252$ & I 4 & I $2^{h} 2 I^{1}$ & $7^{\mathrm{s}} .86$ & $+0^{\circ} 45^{\prime} 23^{\prime \prime} 6$ & $W_{1} 12^{\mathrm{h}} 312$ \\
\hline 2 & $0 \quad 22 \quad 55.33$ & $+74454 \cdot 7$ & $1 / 2($ Schj. I $56+$ Gl. I 24$)$ & 15 & I 28 & 3.50 & +2542.6 & Schj. 4413 \\
\hline 3 & $04741.5^{\circ}$ & +9406.5 & Schj. 3 I I & 16 & I I 54 & 46.35 & +3316.5 & Schj. 4326 \\
\hline 4 & I 643.10 & $+1122 \quad 53.4$ & Yarnall 6 I 3 & I 7 & I I 30 & 9.95 & +132927.4 & Schj. 4193.4 \\
\hline 5 & $\begin{array}{lll}8 & 32 & 28.72\end{array}$ & +21124.0 & $1 / 2\left(W_{2} 8^{h} 757+\right.$ Rü. 2600$)$ & 18 & Ix 22 & 3867 & +133230.3 & Schj. 4152 \\
\hline 6 & $820 \quad 50.72$ & +215022.2 & $W_{2} 8^{h} 44^{2}$ & I9 & I I I 9 & 3.53 & $\begin{array}{r}+133823.4 \\
\end{array}$ & Schj. 4133 \\
\hline 7 & $8 \quad$ I $4 \quad 43.43$ & +221625.9 & $W_{2}^{2} 8^{\mathrm{h}} 284$ & 20 & 2325 & 34.60 & $-44^{2} 54.6$ & Glasg. 62 I 5 \\
\hline 8 & 2943.16 & +104924.4 & $W_{1} 2^{h} 110$ & $2 I$ & 3 I 1 & 6.24 & +512816.4 & AOe. 364 I \\
\hline 9 & 101056.09 & +222 1 3.4 & $W_{1}: 0^{\mathrm{h}} 147$ & 22 & $34^{2}$ & 18.25 & 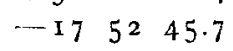 & $\mathrm{AOe}_{2} 2538$ \\
\hline Io & $95^{2} 48.17$ & +52131.6 & Schj. $3^{167}$ & 23 & $\begin{array}{ll}3 & 48\end{array}$ & 55.23 & +3157.2 & Schj. I 212 \\
\hline I I & $\begin{array}{lll}9 & 48 & 6\end{array}$ & +52912.7 & Schj. 3642 & 24 & $\begin{array}{ll}3 & 24\end{array}$ & 22.82 & +271043.1 & $W_{2} 3^{\mathrm{h}} 466$ \\
\hline 12 & $944 \quad$ I $7.0 \mathrm{I}$ & +54527.8 & Schj. 3615 & 25 & 310 & 40.51 & +2628 II.I & $W_{2} 3^{\mathrm{h}} \mathrm{I}_{5}$ \\
\hline I 3 & $1233 \quad 3.3^{6}$ & - & Schj. $4354-5$ & 26 & 644 & 24.05 & +232956.3 & $W_{2} 6^{\mathrm{h}} \geq 27^{\circ}$ \\
\hline
\end{tabular}

Beobachtungen von Planeten am Meridiankreise der Sternwarte in Hamburg.

\begin{tabular}{l|l|l|l|l|l|l|l}
\hline 1885. & M. Z.Hamb. & $\alpha$ app. & Fäd. & d app. & Par. & Beob. - Rechn. & Bemerkungen \\
\hline
\end{tabular}

(1) Ceres.

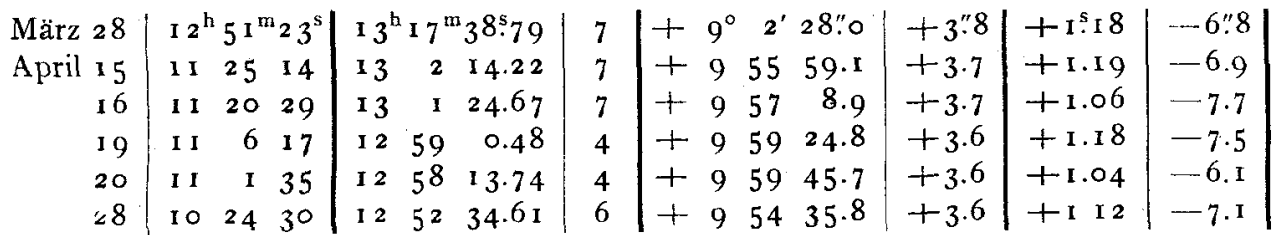

